\title{
MÉTHODES DUALES-HYBRIDES POUR LES ÉQUATIONS DE L'ÉLASTICITÉ LINÉAIRE: UNE APPROCHE UNIFIÉE
}

\author{
MOHAMED FARHLOUL ET MICHEL FORTIN
}

\begin{abstract}
Résumé: On présente une approche unifiée pour la construction d'éléments finis basée sur une formulation duale-hybride des équations de l'élasticité linéaire. Dans cette formulation le tenseur des contraintes est pris en considération mais sa symétrie est relaxée par un multiplicateur de Lagrange qui n'est autre que le rotationnel du déplacement. Cette construction est reliée à l'approximation du problème de Stokes en variables primitives et elle conduit à une nouvelle interprétation de certains éléments finis bien connus et à de nouveaux éléments finis. De plus, toutes les estimations d'erreurs sont valides dans le cas limite incompressible et par conséquent tous ces éléments finis sont applicables au problème de Stokes.
\end{abstract}

Mots-clés : formulation duale-hybride, formulation équilibre, éléments finis mixtes, élasticité linéaire.

Classification mathématique : 65N30 PRIMARY; 73V05 SECONDARY.

\section{INTRODUCTION}

Le problème de l'approximation du tenseur des contraintes dans les équations de l'élasticité linéaire a été soulevé par plusieurs auteurs ces dernières années. La difficulté réside essentiellement dans la symétrie de ce tenseur. Afin d'être plus précis, rappelons les équations de l'élasticité linéaire : Trouver le déplacement $u=\left(u_{1}, u_{2}\right)$ tel que

$$
\begin{cases}-\operatorname{div}(2 \mu \epsilon(u)+\lambda \operatorname{tr}(\epsilon(u)) \delta)=f & \operatorname{dans} \Omega, \\ u=0 & \text { sur } \Gamma,\end{cases}
$$

où $\Omega$ est un ouvert borné de $I R^{2}$ de frontière $\Gamma, f$ est une densité volumique de forces donnée, $\lambda \geq 0$ et $\mu>0$ sont les coefficients de Lamé, $\epsilon(u)$ est le tenseur des déformations donné par

$$
(\epsilon(u))_{i j}=\frac{1}{2}\left(\frac{\partial u_{i}}{\partial x_{j}}+\frac{\partial u_{j}}{\partial x_{i}}\right), \quad i, j=1,2,
$$

$\delta$ est le tenseur identité et pour un tenseur $\tau=\left(\tau_{i j}\right)_{1 \leq i, j \leq 2}, \operatorname{tr}(\tau)=$ $\tau_{11}+\tau_{22}$ (i.e., la trace du tenseur $\tau$ ),

$$
(\operatorname{div} \tau)_{i}=\sum_{j=1}^{2} \frac{\partial \tau_{i j}}{\partial x_{j}}, \quad i=1,2 .
$$

Le but de cet article est de présenter une approche unifiée pour la construction d'éléments finis qui est basée sur une formulation hybride-duale $\mathrm{d} u$ problème (1). De telle formulation provient $\mathrm{d} u$ fait que le problème (1) peut être réécrit comme suit 


$$
\begin{cases}\operatorname{div}(\sigma-p \delta)+f=0 & \operatorname{dans} \Omega, \\ \frac{1}{\lambda} p+\operatorname{div} u=0 & \operatorname{dans} \Omega, \\ \sigma-2 \mu \epsilon(u)=0 & \operatorname{dans} \Omega, \\ u=0 & \text { sur } \Gamma .\end{cases}
$$

Notre construction sera basée sur un résultat bien connu: la construction d'un tenseur symétrique à divergence nulle peut être ramenée à celle d'un vecteur à divergence nulle. En effet, soit

$$
\sigma=\left(\begin{array}{ll}
\sigma_{11} & \sigma_{12} \\
& \\
\sigma_{21} & \sigma_{22}
\end{array}\right)
$$

satisfaisant $\operatorname{div} \sigma=0$. Nous pouvons écrire

$$
\sigma=\left(\begin{array}{ll}
\frac{\partial \psi_{1}}{\partial x_{2}} & -\frac{\partial \psi_{1}}{\partial x_{1}} \\
\frac{\partial \psi_{2}}{\partial x_{2}} & -\frac{\partial \psi_{2}}{\partial x_{1}}
\end{array}\right)
$$

et la symétrie implique

$$
\frac{\partial \psi_{1}}{\partial x_{1}}+\frac{\partial \psi_{2}}{\partial x_{2}}=0
$$

De plus $\sigma \in(H(\operatorname{div} ; \Omega))^{2}$ implique $\psi \in\left(H^{1}(\Omega)\right)^{2}$. En effet, soit $S$ une interface entre $\Omega_{1}$ et $\Omega_{2}$. On a donc $(\sigma n)_{i}=\frac{\partial \psi_{i}}{\partial t}, i=1,2$, et la continuité de la trace normale du tenseur des contraintes est équivalente à celle de la dérivée tangentielle de $\psi$. Mais maintenant, construire des approximations d'une fonction dans $H^{1}$ à divergence nulle est un problème bien connu qui a été largement étudié pour l'approximation du problème de Stokes et de Navier-Stokes et il existe tout un catalogue de telles approximations. Notre intention est d'exploiter ce catalogue dans le but d'obtenir de nouveaux résultats ou de simplifier considérablement ceux existants dans la littérature.

Pour nous amener au cas où div $\sigma=0$, nous introduisons une formulation hybride-duale de (2) qui est basée sur la dualisation de la contrainte de symétrie et la continuité de la trace normale du tenseur $\sigma-p \delta$ à travers les interéléments de la triangulation de $\bar{\Omega}$. Nous présenterons une approche unifiée pour construire des éléments finis qui est basée sur de telle formulation, c'est le résultat principal de cet article. Nous notons aussi que par cette approche nous obtenons des nouveaux éléments finis pour lesquels des estimations d'erreurs optimales seront données.

Mentionnons que toutes les estimations, qui seront déduites pour tous les éléments finis étudiés par la suite pour le problème de l'élasticité linéaire, sont indépendantes $d u$ coefficient $\lambda$. Par conséquent tous ces éléments finis sont applicables au problème de Stokes. En effet, les équations de Stokes peuvent être formulées comme suit 


$$
\begin{cases}-2 \nu \operatorname{div} \epsilon(u)+\nabla p=f & \operatorname{dans} \Omega, \\ \operatorname{div} u=0 & \operatorname{dans} \Omega, \\ u=0 & \text { sur } \Gamma,\end{cases}
$$

où $u=\left(u_{1}, u_{2}\right)$ est le champ de vitesse, $p$ est la pression et $\nu>0$ est la viscosité. En introduisant $\sigma=2 \nu \epsilon(u)$ comme variable auxiliaire, le problème (3) peut être écrit sous la forme

$$
\begin{cases}\operatorname{div}(\sigma-p \delta)+f=0 & \operatorname{dans} \Omega, \\ \operatorname{div} u=0 & \operatorname{dans} \Omega, \\ \sigma-2 \nu \epsilon(u)=0 & \operatorname{dans} \Omega, \\ u=0 & \text { sur } \Gamma .\end{cases}
$$

Alors le problème de Stokes peut être considéré comme une limite du problème de l'élasticité (2) lorsque $\lambda \rightarrow+\infty$.

\section{UNE MÉTHODE VARIATIONNELLE DUALE-HYBRIDE}

Soit $T_{h}$ une triangulation de $\bar{\Omega}$ formée de triangles $K$ ( $\Gamma$ est supposée polygonale). Si $v$ est une fonction définie sur $\Omega, v^{K}$ désignera sa restriction à l'élément $K$. Soit $p$ un nombre réel et $q$ son conjugué tel que $4 / 3<p<2$. Nous définissons les espaces suivants:

$$
\begin{gathered}
\Sigma=\left\{(\tau, q) \in\left(L^{q}(\Omega)\right)^{4} \times\left(L^{q}(\Omega) \cap L_{0}^{2}(\Omega)\right) ;\right. \\
\left.\left(\tau^{K}-q^{K} \delta\right) \in\left(W_{q}(\operatorname{div} ; K)\right)^{2} \forall K \in T_{h}\right\} \\
\Lambda=\left\{\mu \in \prod_{K \in T_{h}} W^{1 / q, p}(\partial K), \mu^{K_{1}}=\mu^{K_{2}} \operatorname{sur} \partial K_{1} \cap \partial K_{2},\right. \\
\left.\forall K_{1}, K_{2} \in T_{h}, \mu^{K}=0 \operatorname{sur} \partial K \cap \Gamma, \forall K \in T_{h}\right\} \\
M=\left(L^{q}(\Omega)\right)^{2} \times(\Lambda)^{2} \times L^{q}(\Omega)
\end{gathered}
$$

où

$$
\begin{gathered}
W_{q}(\operatorname{div} ; K)=\left\{v \in\left(L^{q}(K)\right)^{2} ; \operatorname{div} v \in L^{q}(K)\right\}, \\
L_{0}^{2}(\Omega)=\left\{q \in L^{2}(\Omega) ; \int_{\Omega} q d x=0\right\}
\end{gathered}
$$

et $W^{1 / q, p}(\partial K)$ est l'espace des traces sur la frontière de $K$ des fonctions de $W^{1, p}(K)$.

Pour un tenseur $\tau=\left(\tau_{i j}\right)_{1 \leq i, j \leq 2}$, on définit sa trace normale par $\tau n=$ $\left(\tau_{1} \cdot n, \tau_{2} \cdot n\right)$, où $\tau_{i}=\left(\tau_{i 1}, \tau_{i 2}\right), i=1,2$ et $n$ désigne la normale unitaire à $\Gamma$ dirigée vers l'extérieur de $\Omega$. Les composantes normale et tangentielle, $\tau_{n n}$ et $\tau_{n t}$, du vecteur $\tau n$, sont données par $\tau_{n n}=\tau n \cdot n$ et $\tau_{n t}=\tau n \cdot t$, où $t$ désigne la tangente unitaire à $\Gamma$. Nous introduisons aussi les formes 
bilinéaires continues $a(.,$.$) et b(.,$.$) définies respectivement sur \Sigma \times \Sigma, \Sigma \times M$, données par

$$
\begin{aligned}
& a((\sigma, p) ;(\tau, q))=\frac{1}{2 \mu} \int_{\Omega} \sigma: \tau d x+\frac{1}{\lambda} \int_{\Omega} p q d x \\
& \left\{\begin{aligned}
b\left((\tau, q) ;\left(v, \mu_{n}, \mu_{t}, \theta\right)\right)= & \sum_{K \in T_{h}} \int_{K} \operatorname{div}(\tau-q \delta) \cdot v d x \\
& -\sum_{K \in T_{h}} \int_{\partial K}(\tau-q \delta)_{n n} \mu_{n} d s
\end{aligned}\right. \\
& -\sum_{K \in T_{h}} \int_{\partial K} \tau_{n t} \mu_{t} d s+\int_{\Omega} a s(\tau) \theta d x
\end{aligned}
$$

où $\sigma: \tau=\sum_{i, j=1}^{2} \sigma_{i j} \tau_{i j}$ et $a s(\tau)=\tau_{21}-\tau_{12}$.

La formulation duale-hybride des équations d'élasticité (2) est donnée par: Trouver $(\sigma, p) \in \Sigma$ et $\left(u, \lambda_{n}, \lambda_{t}, \omega\right) \in M$ tels que

$$
\begin{cases}a((\sigma, p) ;(\tau, q))+b\left((\tau, q) ;\left(u, \lambda_{n}, \lambda_{t}, \omega\right)\right)=0 & \forall(\tau, q) \in \Sigma \\ b\left((\sigma, p) ;\left(v, \mu_{n}, \mu_{t}, \theta\right)\right)+(f, v)=0 & \forall\left(v, \mu_{n}, \mu_{t}, \theta\right) \in M\end{cases}
$$

où $(f, v)=\int_{\Omega} f \cdot v d x$.

Mentionnons que si la solution $u$ du problème d'élsticité (1) satisfait $p=$ $-\lambda \operatorname{div} u \in L^{q}(\Omega) \cap L_{0}^{2}(\Omega)$ et $(\sigma-p \delta) \in\left(W_{q}(\operatorname{div} ; \Omega)\right)^{2}$, où $\sigma=2 \mu \epsilon(u)$, alors (voir $[6])\left((\sigma, p) ;\left(u, u \cdot n, u \cdot t, \frac{1}{2} \operatorname{rot} u\right)\right)$ est l'unique solution de $(10)$, où $\operatorname{rot} u=\partial u_{2} / \partial x_{1}-\partial u_{1} / \partial x_{2}$.

Dans le but de donner une approche unifiée de la construction d'éléments finis mixtes pour le problème (10), nous donnons une formulation équilibre qui est équivalente à la formulation (10).

Soit $\Sigma^{f}$ la variété affine définie par

$$
\Sigma^{f}=\left\{(\tau, q) \in \Sigma ; \operatorname{div}\left(\tau^{K}-q^{K} \delta\right)+f^{K}=0 \text { dans } K, \forall K \in T_{h}\right\},
$$

$\Sigma^{0}$ son sous-espace associé et $X=(\Lambda)^{2} \times L^{q}(\Omega)$, où $\Lambda$ est défini par (6). Nous introduisons la forme bilinéaire continue $b_{1}(.,$.$) définie sur \Sigma \times X$ et donnée par

$$
\left\{\begin{aligned}
b_{1}\left((\tau, q) ;\left(\mu_{n}, \mu_{t}, \theta\right)\right)= & -\sum_{K \in T_{h}} \int_{\partial K}(\tau-q \delta)_{n n} \mu_{n} d s \\
& -\sum_{K \in T_{h}} \int_{\partial K} \tau_{n t} \mu_{t} d s+\int_{\Omega} a s(\tau) \theta d x
\end{aligned}\right.
$$

La formulation équilibre est définie comme suit : Trouver $(\sigma, p) \in \Sigma^{f}$ et $\left(\lambda_{n}, \lambda_{t}, \omega\right) \in X$ tels que

$$
\begin{cases}a((\sigma, p) ;(\tau, q))+b_{1}\left((\tau, q) ;\left(\lambda_{n}, \lambda_{t}, \omega\right)\right)=0 & \forall(\tau, q) \in \Sigma^{0} \\ b_{1}\left((\sigma, p) ;\left(\mu_{n}, \mu_{t}, \theta\right)\right)=0 & \forall\left(\mu_{n}, \mu_{t}, \theta\right) \in X .\end{cases}
$$

La construction d'éléments finis pour le problème (13) est basée sur l'idée principale suivante: Soit $(\widehat{\sigma}, \widehat{p})$ un élément arbitraire de $\Sigma^{f}$, on peut écrire $\sigma-p \delta=\left(\sigma^{0}-p^{0} \delta\right)+(\widehat{\sigma}-\widehat{p} \delta)$ avec $\operatorname{div}\left(\sigma^{0}-p^{0} \delta\right)=0$ sur chaque $K \in T_{h}$. 
Maintenant, en écrivant $\sigma^{0}-p^{0} \delta=\left(\sigma_{1}^{0}, \sigma_{2}^{0}\right)$, on a div $\sigma_{i}^{0}=0, i=1,2$, sur chaque $K \in T_{h}$. Alors, il existe $\left(\psi_{1}, \psi_{2}\right)$ tels que $\sigma_{i}=\operatorname{rot} \psi_{i}, i=1$, 2 , i.e.,

$$
\sigma^{0}-p^{0} \delta=\left(\begin{array}{ll}
\frac{\partial \psi_{1}}{\partial x_{2}} & -\frac{\partial \psi_{1}}{\partial x_{1}} \\
\frac{\partial \psi_{2}}{\partial x_{2}} & -\frac{\partial \psi_{2}}{\partial x_{1}}
\end{array}\right)
$$

D'autre part, puisque $\int_{\Omega} a s\left(\sigma^{0}\right) \theta d x=0$ pour tout $\theta \in L^{q}(\Omega)$ (en prenant en considération que le choix de $\widehat{\sigma}$ est fait de telle sorte que $\int_{\Omega} a s(\widehat{\sigma}) \theta d x=0$ pour tout $\theta \in L^{q}(\Omega)$ ), on a $\int_{\Omega} \operatorname{div} \psi \theta d x=0$ pour tout $\theta \in L^{q}(\Omega)$, où $\psi=\left(\psi_{1}, \psi_{2}\right)$. Donc, la construction de l'approximation du tenseur $\sigma$ sera donnée par celle du vecteur $\psi$ telle que $\int_{\Omega} \operatorname{div} \psi \theta d x=0$, ce qui est reliée à l'approximation du problème de Stokes en variables primitives, i.e., le problème (3). Plus précisement, l'espace d'approximation pour la vitesse, respectivement pour la pression, donnera celui pour $\psi$, respectivement pour $\omega$.

Puisqu'il y a plusieurs exemples de discrétisations stables du problème de Stokes en variables primitives (voir Girault et Raviart [8] et Brezzi et Fortin [3]), nous donnerons quelques uns correspondants pour le problème (13) et aussi pour le problème (10).

\section{DISCRÉTISATION}

Soit $\Sigma_{h} \subset \Sigma, W_{h} \subset\left(L^{q}(\Omega)\right)^{2}, \Lambda_{h} \subset \Lambda$ et $Q_{h} \subset L^{q}(\Omega)$ des espaces de dimension finie (on suppose que $\Sigma_{h}$ contient les tenseurs constants), et posons $M_{h}=W_{h} \times\left(\Lambda_{h}\right)^{2} \times Q_{h}$. Alors, le problème (10) est discrétisé par le suivant: Trouver $\left(\sigma_{h}, p_{h}\right) \in \Sigma_{h}$ et $\left(u_{h}, \lambda_{n h}, \lambda_{t h}, \omega_{h}\right) \in M_{h}$ tels que

$$
\left\{\begin{array}{c}
a\left(\left(\sigma_{h}, p_{h}\right) ;\left(\tau_{h}, q_{h}\right)\right)+b\left(\left(\tau_{h}, q_{h}\right),\left(u_{h}, \lambda_{n h}, \lambda_{t h}, \omega_{h}\right)\right)=0 \\
\forall\left(\tau_{h}, q_{h}\right) \in \Sigma_{h}, \\
b\left(\left(\sigma_{h}, p_{h}\right) ;\left(v_{h}, \mu_{n h}, \mu_{t h}, \theta_{h}\right)\right)+\left(f, v_{h}\right)=0 \\
\forall\left(v_{h}, \mu_{n h}, \mu_{t h}, \theta_{h}\right) \in M_{h} .
\end{array}\right.
$$

Le problème discret de (13) est comme suit: Trouver $\left(\sigma_{h}, p_{h}\right) \in \Sigma_{h}^{f}$ et $\left(\lambda_{n h}, \lambda_{t h}, \omega_{h}\right) \in X_{h}$ tels que

$$
\left\{\begin{array}{c}
a\left(\left(\sigma_{h}, p_{h}\right) ;\left(\tau_{h}, q_{h}\right)\right)+b_{1}\left(\left(\tau_{h}, q_{h}\right),\left(\lambda_{n h}, \lambda_{t h}, \omega_{h}\right)\right)=0 \\
\forall\left(\tau_{h}, q_{h}\right) \in \Sigma_{h}^{0}, \\
b_{1}\left(\left(\sigma_{h}, p_{h}\right) ;\left(\mu_{n h}, \mu_{t h}, \theta_{h}\right)\right)=0 \\
\forall\left(\mu_{n h}, \mu_{t h}, \theta_{h}\right) \in X_{h},
\end{array}\right.
$$

où $\Sigma_{h}^{0} \subset \Sigma^{0}, X_{h}=\left(\Lambda_{h}\right)^{2} \times Q_{h} \subset X$ sont des espaces de dimension finie et $\Sigma_{h}^{f}=\left(\widehat{\sigma}_{h}, \widehat{p}_{h}\right)+\Sigma_{h}^{0}$. Le tenseur $\left(\widehat{\sigma}_{h}, \widehat{p}_{h}\right)$ est un élément de $\Sigma$ tel que $\operatorname{div}\left(\widehat{\sigma}_{h}^{K}-\widehat{p}_{h}^{K} \delta\right)+f_{h}^{K}=0$ sur chaque $K, f_{h}$ étant une interpolation appropriée de $f$ (nous référons à [1] et [12] pour de telle construction).

Avant de donner quelques exemples des espaces $\Sigma_{h}, M_{h}$ et $\Sigma_{h}^{0}$, nous allons tout d'abord donner un résultat abstrait (voir [6] pour la preuve), concernant la condition inf-sup, sous les deux hypothèses suivantes: 
$(H 1)\left\{\begin{array}{l}\text { Soit } w_{h} \in W_{h} \text { donné. Alors, il existe } \widehat{\tau}_{h} \in\left(W_{q}(\operatorname{div} ; \Omega)\right)^{2} \text { tel que } \\ \left(\widehat{\tau}_{h}, 0\right) \in \Sigma_{h}, \operatorname{div} \widehat{\tau}_{h}=w_{h} \text { dans } \Omega \text { et }\left\|\widehat{\tau}_{h}\right\|_{0, \Omega} \leq C\left\|w_{h}\right\|_{0, \Omega},\end{array}\right.$ où $C$ est une constante indépendante de $h$,

$(H 2)\left\{\begin{array}{l}\text { Il existe un espace de dimension finie } V_{h} \subset\left(H_{0}^{1}(\Omega)\right)^{2} \text { tel que } \\ \text { la discrétisation du problème de Stokes }(3) \operatorname{par}\left(V_{h}, Q_{h}\right) \text { est stable } \\ \text { et rot } V_{h} \subset \Sigma_{h}^{0},\end{array}\right.$ où $\operatorname{rot} V_{h}=\left\{\left(\operatorname{rot} v_{1}, \operatorname{rot} v_{2}\right),\left(v_{1}, v_{2}\right) \in V_{h}\right\}$.

Proposition 1. Sous les hypothèses $(H 1)$ et $(H 2)$, il existe une constante $C$ indépendante de $h$ telle que

$$
\begin{array}{ll}
\sup _{\left(\tau_{h}, q_{h}\right) \in \Sigma_{h}} \frac{b\left(\left(\tau_{h}, q_{h}\right) ;\left(v_{h}, \lambda_{n h}, \lambda_{t h}, \theta_{h}\right)\right)}{\left\|\left(\tau_{h}, q_{h}\right)\right\|_{\tilde{\Sigma}}} \geq & C\left(\left\|v_{h}\right\|_{0, \Omega}+\left\|\theta_{h}\right\|_{0, \Omega}\right) \\
& \forall\left(v_{h}, \lambda_{n h}, \lambda_{t h}, \theta_{h}\right) \in M_{h} \\
\text { ò̀ }\left\|\left(\tau_{h}, q_{h}\right)\right\|_{\tilde{\Sigma}}=\left(\left\|\tau_{h}\right\|_{0, \Omega}^{2}+\left\|q_{h}\right\|_{0, \Omega}^{2}\right)^{1 / 2} &
\end{array}
$$

Grâce à la condition inf-sup (16), l'analyse des éléments finis que nous allons introduire pour le problème (14) (et par conséquent pour le problème (15)) est similaire à celle donnée dans [5]. La seule partie que nous devons vérifier, pour obtenir des estimations d'erreurs optimales indépendantes du coefficient $\lambda$, est le résultat suivant.

Proposition 2. Il existe une constante $\alpha$, indépendante de $h$ et de $\lambda$, telle que

$$
a\left(\left(\tau_{h}, q_{h}\right) ;\left(\tau_{h}, q_{h}\right)\right) \geq \alpha\left\|\left(\tau_{h}, q_{h}\right)\right\|_{\tilde{\Sigma}}, \forall\left(\tau_{h}, q_{h}\right) \in Z_{h},
$$

où

$$
\begin{array}{r}
Z_{h}=\left\{\left(\tau_{h}, q_{h}\right) \in \Sigma_{h} ; \quad b\left(\left(\tau_{h}, q_{h}\right) ;\left(v_{h}, \mu_{n h}, \mu_{t h}, \theta_{h}\right)\right)=0\right. \\
\left.\forall\left(v_{h}, \mu_{n h}, \mu_{t h}, \theta_{h}\right) \in M_{h}\right\} .
\end{array}
$$

La preuve de ce dernier résultat, pour tous les éléments finis que nous allons présenter, est la même que celle de la Proposition 3.2 dans [5].

Nous sommes maintenant en position de présenter quelques éléments finis pour le problème (14), aussi pour le problème (15). Auparavant, précisons quelques notations. Nous notons par $P_{k}(K)$ l'espace des polynômes de degré inférieur ou égal à $k$. L'espace $R T_{k}(K)$ est donné par

$$
R T_{k}(K)=\left(P_{k}(K)\right)^{2}+x P_{k}(K)
$$

où $x=\left(x_{1}, x_{2}\right)$, qui n'est autre que l'élément fini de Raviart-Thomas d'ordre $k$ (voir [10]). Nous définissons a usssi

$$
R_{k}(\partial K)=\left\{\phi ; \phi \in L^{2}(\partial K),\left.\phi\right|_{e} \in P_{k}(e) \forall e \in \partial K\right\} .
$$

Finalement, nous notons par $b_{K}$ la "fonction bulle" définie par

$$
b_{K}(x)=\lambda_{1}(x) \lambda_{2}(x) \lambda_{3}(x),
$$

où $\lambda_{1}, \lambda_{2}, \lambda_{3}$ sont les coordonnées barycentriques dans $K$. 
Mentionnons que, pour tous les éléments finis que nous allons présenter, les hypothèses $(H 1)$ et $(H 2)$ sont satisfaites par construction.

ExEmple 3. Notre premier exemple est de considérer une approximation classique du problème de Stokes par ce qu'on appelle le "Mini-élément" (voir [3]), i.e., la vitesse, respectivement la pression, est approximée par $\left(P_{1}(K) \oplus b_{K}\right)^{2}$ continue, respectivement $P_{1}(K)$ continue. Alors, les espaces $\Sigma_{h}^{0}$ et $X_{h}$ correspondants sont donnés par

$$
\begin{gathered}
\Sigma_{h}^{0}=\left\{\begin{array}{l}
(\tau, q) \in \Sigma ; q^{K} \in P_{0}(K), \\
\tau^{K}-q^{K} \delta=\left(\operatorname{rot}\left(w_{1}+\alpha_{1} b_{K}\right), \operatorname{rot}\left(w_{2}+\alpha_{2} b_{K}\right)\right) \\
\left.\left(w_{1}, w_{2}\right) \in\left(P_{1}(K)\right)^{2},\left(\alpha_{1}, \alpha_{2}\right) \in\left(P_{0}(K)\right)^{2}, \forall K \in T_{h}\right\}
\end{array}\right. \\
X_{h}=\left\{\left(\mu_{n}, \mu_{t}, \theta\right) \in X ;\left(\mu_{n}^{K}, \mu_{t}^{K}\right) \begin{array}{l}
\in\left(R_{0}(\partial K)\right)^{2}, \theta \in C^{0}(\bar{\Omega}) \\
\left.\theta^{K} \in P_{1}(K), \forall K \in T_{h}\right\}
\end{array}\right.
\end{gathered}
$$

Puisque $\left(\operatorname{rot} b_{K}\right) \cdot n=0$ sur chaque côté de $K$, on peut écrire $\tau^{K}-q^{K} \delta=$ $\left(\operatorname{rot} w_{1}, \operatorname{rot} w_{2}\right)+\left(\alpha_{1} \operatorname{rot} b_{K}, \alpha_{2} \operatorname{rot} b_{K}\right)$ et donc seulement la première partie de ce tenseur qui sera modifiée pour obtenir l'approximation correspondante pour le problème (14). Plus présisement, nous avons

$$
\begin{aligned}
& \Sigma_{h}=\left\{(\tau, q) \in \Sigma ; q^{K} \in P_{0}(K),\right. \\
& \tau^{K}-q^{K} \delta \in\left(R T_{0}(K)\right)^{2}+\left(\alpha_{1} \operatorname{rot} b_{K}, \alpha_{2} \operatorname{rot} b_{K}\right), \\
& \left.\left(\alpha_{1}, \alpha_{2}\right) \in\left(P_{0}(K)\right)^{2}, \forall K \in T_{h}\right\}, \\
& M_{h}=\left\{\left(v, \mu_{n}, \mu_{t}, \theta\right) \in M ; \quad v^{K} \in\left(P_{0}(K)\right)^{2},\left(\mu_{n}^{K}, \mu_{t}^{K}\right) \in\left(R_{0}(\partial K)\right)^{2},\right. \\
& \left.\theta \in C^{0}(\bar{\Omega}), \theta^{K} \in P_{1}(K), \forall K \in T_{h}\right\} \text {. }
\end{aligned}
$$

Comme degrés de liberté pour cet élément fini, nous avons

$$
\begin{aligned}
& \left\{\begin{array}{l}
\int_{K} p p_{0} d x, \forall p_{0} \in P_{0}(K), \\
\int_{\partial K}(\sigma-p \delta) n \cdot p_{0} d s, \forall p_{0} \in\left(R_{0}(\partial K)\right)^{2}, \\
\int_{\Omega} a s(\sigma) p_{1} d x, \forall p_{1} \in C^{0}(\bar{\Omega}),\left.p_{1}\right|_{K} \in P_{1}(K), \forall K \in T_{h},
\end{array}\right. \\
& \left\{\begin{array}{l}
\int_{K} u \cdot p_{0} d x, \forall p_{0} \in\left(P_{0}(K)\right)^{2}, \\
\int_{\partial K} \lambda_{n} p_{0} d s, \forall p_{0} \in R_{0}(\partial K), \\
\int_{\partial K} \lambda_{t} p_{0} d s, \forall p_{0} \in R_{0}(\partial K), \\
\omega \in\left\{\theta \in C^{0}(\bar{\Omega}),\left.\theta\right|_{K} \in P_{1}(K), \forall K \in T_{h}\right\} .
\end{array}\right.
\end{aligned}
$$

Cet élément fini n'est autre que l'élément PEERS d'Arnold-Brezzi- Douglas [2] avec relaxation de la continuité de la trace normale de $(\tau-q \delta) n$ à travers les interéléments de la triangulation de $\bar{\Omega}$. L'analyse de cet élément fini et les estimations d'erreurs optimales $(O(h))$ pour le tenseur $\sigma$ et le déplacement $u$ sont données dans [2] et aussi dans [11]. Pour obtenir des estimations d'erreurs optimales pour les multiplicateurs de Lagrange $\lambda_{n}$ et $\lambda_{t}$ on peut suivre la procédure donnée dans [5]. 
Remarque 4. Pour ce premier exemple, $(H 1)$ est une conséquence du fait que pour tout $w_{h} \in\left\{v \in\left(L^{q}(\Omega)\right)^{2} ; v^{K} \in\left(P_{0}(K)\right)^{2}, \forall K \in T_{h}\right\}$, il existe $\widehat{\tau}_{h} \in\left(W_{q}(\operatorname{div} ; \Omega)\right)^{2}$ tel que $\widehat{\tau}_{h}^{K} \in\left(R T_{0}(K)\right)^{2}$ pour tout $K \in T_{h}$, div $\widehat{\tau}_{h}=w_{h}$ dans $\Omega$, et $\left\|\widehat{\tau}_{h}\right\|_{0, \Omega} \leq C\left\|w_{h}\right\|_{0, \Omega}$. Tandis que (H2) n'est autre que la stabilité de l'approximation du problème de Stokes (3) par le "Mini-élément". Alors, en utilisant la Proposition 1, nous obtenons une preuve simple de la stabilité de l'élément PEERS.

Exemple 5. Cet exemple concerne l'approximation de (3) par l'élément de Hood-Taylor [9], i.e., la vitesse, respectivement la pression, est approximée par $\left(P_{2}(K)\right)^{2}$ continue, respectivement $P_{1}(K)$ continue. Les espaces $\Sigma_{h}^{0}$ et $X_{h}$ correspondants pour le problème (15) sont donnés par

$$
\begin{gathered}
\Sigma_{h}^{0}=\left\{\begin{aligned}
(\tau, q) \in \Sigma ; & q^{K} \in P_{1}(K), \tau^{K}-q^{K} \delta=\left(\operatorname{rot} w_{1}, \operatorname{rot} w_{2}\right), \\
\left.\left(w_{1}, w_{2}\right) \in\left(P_{2}(K)\right)^{2}, \forall K \in T_{h}\right\}, & \\
X_{h}=\left\{\left(\mu_{n}, \mu_{t}, \theta\right) \in X ;\left(\mu_{n}^{K}, \mu_{t}^{K}\right) \quad\right. & \in\left(R_{1}(\partial K)\right)^{2}, \theta \in C^{0}(\bar{\Omega}), \\
& \left.\theta^{K} \in P_{1}(K), \forall K \in T_{h}\right\} .
\end{aligned}\right.
\end{gathered}
$$

L'élément fini correspondant à ce dernier pour le problème (14) est donné par

$$
\begin{gathered}
\Sigma_{h}=\left\{(\tau, q) \in \Sigma ; q^{K} \in P_{1}(K), \tau^{K}-q^{K} \delta \in\left(R T_{1}(K)\right)^{2}, \forall K \in T_{h}\right\}, \\
M_{h}=\left\{\left(v, \mu_{n}, \mu_{t}, \theta\right) \in M ; \quad v^{K} \in\left(P_{1}(K)\right)^{2},\left(\mu_{n}^{K}, \mu_{t}^{K}\right) \in\left(R_{1}(\partial K)\right)^{2},\right. \\
\left.\theta \in C^{0}(\bar{\Omega}), \theta^{K} \in P_{1}(K), \forall K \in T_{h}\right\} .
\end{gathered}
$$

Les degrés de liberté pour l'élément fini (24)-(25) sont

$$
\begin{aligned}
& \left\{\begin{array}{l}
\int_{K} p p_{1} d x, \forall p_{1} \in P_{1}(K), \\
\int_{\partial K}(\sigma-p \delta) n \cdot p_{1} d s, \forall p_{1} \in\left(R_{1}(\partial K)\right)^{2}, \\
\int_{\Omega}(\sigma-p \delta): p_{0} d x, \forall p_{0} \in\left(P_{0}(K)\right)^{4},
\end{array}\right. \\
& \left\{\begin{array}{l}
\int_{K} u \cdot p_{1} d x, \forall p_{1} \in\left(P_{1}(K)\right)^{2}, \\
\int_{\partial K} \lambda_{n} p_{1} d s, \forall p_{1} \in R_{1}(\partial K), \\
\int_{\partial K} \lambda_{t} p_{1} d s, \forall p_{1} \in R_{1}(\partial K), \\
\omega \in\left\{\theta \in C^{0}(\bar{\Omega}),\left.\theta\right|_{K} \in P_{1}(K), \forall K \in T_{h}\right\} .
\end{array}\right.
\end{aligned}
$$

En suivant la procédure dans [5], nous optenons des estimations d'erreurs optimales $\left(O\left(h^{2}\right)\right)$ pour ces deux derniers éléments finis.

Exemple 6. Maintenant, considérons l'approximation de (3) par l'élément de Crouzeix-Raviart [4], i.e., la vitesse, respectivement la pression, est approximée par $\left(P_{2}(K) \oplus b_{K}\right)^{2}$ continue, respectivement $P_{1}(K)$ discontinue. Les espaces $\Sigma_{h}^{0}$ et $X_{h}$ correspondants sont donnés par 


$$
\begin{aligned}
\Sigma_{h}^{0}=\{\quad(\tau, q) & \in \Sigma ; q^{K} \in P_{1}(K), \\
& \tau^{K}-q^{K} \delta=\left(\operatorname{rot}\left(w_{1}+\alpha_{1} b_{K}\right), \operatorname{rot}\left(w_{2}+\alpha_{2} b_{K}\right)\right), \\
& \left.\left(w_{1}, w_{2}\right) \in\left(P_{2}(K)\right)^{2},\left(\alpha_{1}, \alpha_{2}\right) \in\left(P_{0}(K)\right)^{2}, \forall K \in T_{h}\right\},
\end{aligned}
$$

$X_{h}=\left\{\left(\mu_{n}, \mu_{t}, \theta\right) \in X ;\left(\mu_{n}^{K}, \mu_{t}^{K}\right) \in\left(R_{1}(\partial K)\right)^{2}, \theta^{K} \in P_{1}(K), \forall K \in T_{h}\right\}$.

Cet élément fini n'est autre que celui du plus bas degré d'Amara-Thomas [1] qui converge en $O\left(h^{2}\right)$.

L'élément fini correspondant pour le problème (14) est donné par

$$
\begin{gathered}
\Sigma_{h}=\left\{\begin{aligned}
(\tau, q) \in \Sigma ; & q^{K} \in P_{1}(K), \\
\tau^{K}-q^{K} \delta \in & \left(R T_{1}(K)\right)^{2}+\left(\alpha_{1} \operatorname{rot} b_{K}, \alpha_{2} \operatorname{rot} b_{K}\right), \\
& \left.\left(\alpha_{1}, \alpha_{2}\right) \in\left(P_{0}(K)\right)^{2}, \forall K \in T_{h}\right\},
\end{aligned}\right. \\
\begin{aligned}
& M_{h}=\left\{\left(v, \mu_{n}, \mu_{t}, \theta\right) \in M ; v^{K} \in\left(P_{1}(K)\right)^{2},\right.\left(\mu_{n}^{K}, \mu_{t}^{K}\right) \in\left(R_{1}(\partial K)\right)^{2}, \\
&\left.\theta^{K} \in P_{1}(K), \forall K \in T_{h}\right\} .
\end{aligned}
\end{gathered}
$$

Les degrés de liberté pour cet élément fini sont

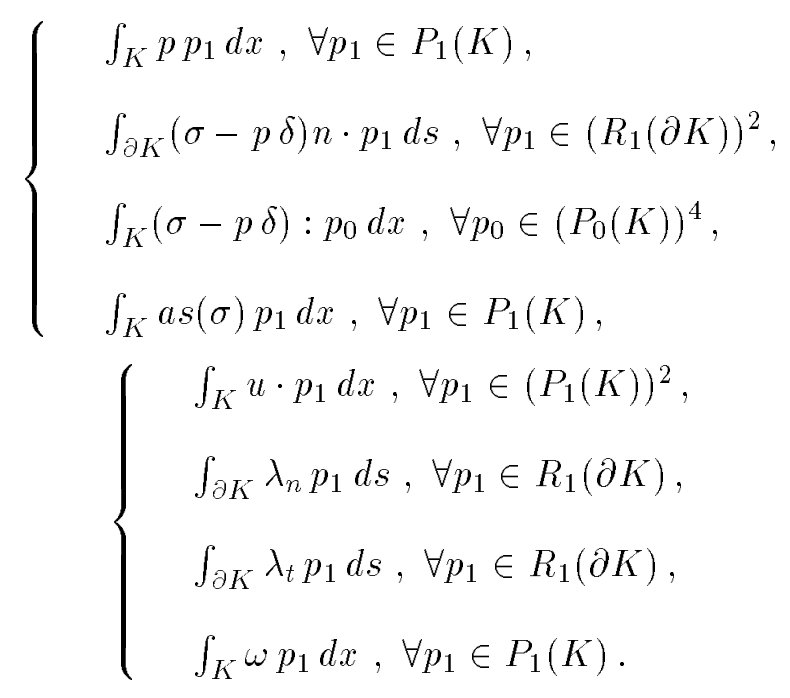

EXemple 7. Le dernier exemple que nous considérons est celui concernant l'approximation du problème de Stokes (3) par $\left(P_{1}(K)\right)^{2} \oplus \operatorname{span}\left\{p^{1}, p^{2}, p^{3}\right\}$ pour la vitesse et $P_{0}(K)$ pour la pression, où $p^{1}=n^{1} \lambda_{2} \lambda_{3}, p^{2}=n^{2} \lambda_{3} \lambda_{1}$ et $p^{3}=n^{3} \lambda_{1} \lambda_{2}\left(\lambda_{i}, i=1,2,3\right.$, étant les coordonnées barycentriques dans $K$ et $n^{i}$ désigne la normale unitaire au côté $e_{i}$ dirigée vers l'extérieur de $K$, $i=1,2,3)$. Les espaces $\Sigma_{h}^{0}$ et $X_{h}$ correspondants pour le problème (15) sont donnés par

$$
\begin{aligned}
\Sigma_{h}^{0}=\{(\tau, q) & \in \Sigma ; q^{K} \in P_{0}(K), \\
& \tau^{K}-q^{K} \delta=\left(\operatorname{rot} w_{1}, \operatorname{rot} w_{2}\right)+\sum_{i=1}^{3} \alpha_{i}\left(\operatorname{rot} p_{1}^{i}, \operatorname{rot} p_{2}^{i}\right), \\
& \left.\left(w_{1}, w_{2}\right) \in\left(P_{1}(K)\right)^{2}, \alpha_{i} \in P_{0}(K), 1 \leq i \leq 3, \forall K \in T_{h}\right\}
\end{aligned}
$$




$$
\begin{aligned}
& X_{h}=\left\{\left(\mu_{n}, \mu_{t}, \theta\right) \in X ; \mu_{n}^{K} \in R_{1}(\partial K), \mu_{t}^{K} \in R_{0}(\partial K),\right. \\
& \left.\theta^{K} \in P_{0}(K), \forall K \in T_{h}\right\} \text {, }
\end{aligned}
$$

où $p^{i}=\left(p_{1}^{i}, p_{2}^{i}\right), i=1,2,3$.

L'élément fini correspondant à ce dernier pour le problème (14) est donné par

$$
\begin{gathered}
\Sigma_{h}=\left\{\begin{array}{r}
(\tau, q) \in \Sigma ; q^{K} \in P_{0}(K), \\
\tau^{K}-q^{K} \delta \in\left(R T_{0}(K)\right)^{2}+\sum_{i=1}^{3} \alpha_{i}\left(\operatorname{rot} p_{1}^{i}, \operatorname{rot} p_{2}^{i}\right), \\
\left.\left(\alpha_{1}, \alpha_{2}, \alpha_{3}\right) \in\left(P_{0}(K)\right)^{3}, \forall K \in T_{h}\right\},
\end{array}\right. \\
M_{h}=\left\{\begin{array}{r}
\left(v, \mu_{n}, \mu_{t}, \theta\right) \in M ; v^{K} \in\left(P_{0}(K)\right)^{2}, \mu_{n}^{K} \in R_{1}(\partial K), \\
\left.\mu_{t}^{K} \in R_{0}(\partial K), \theta^{K} \in P_{0}(K), \forall K \in T_{h}\right\} .
\end{array}\right.
\end{gathered}
$$

Les degrés de liberté pour ce dernier élément fini sont

$$
\begin{aligned}
& \left\{\begin{array}{l}
\int_{K} p p_{0} d x, \forall p_{0} \in P_{0}(K), \\
\int_{\partial K}(\sigma-p \delta)_{n n} p_{1} d s, \forall p_{1} \in R_{1}(\partial K), \\
\int_{\partial K} \sigma_{n t} p_{0} d s, \forall p_{0} \in \in R_{0}(\partial K),
\end{array}\right. \\
& \left\{\begin{array}{l}
\int_{K} u \cdot p_{0} d x, \forall p_{0} \in\left(P_{0}(K)\right)^{2} \\
\int_{\partial K} \lambda_{n} p_{1} d s, \forall p_{1} \in R_{1}(\partial K) \\
\int_{\partial K} \lambda_{t} p_{0} d s, \forall p_{0} \in R_{0}(\partial K) \\
\int_{K} \omega p_{0} d x, \forall p_{0} \in P_{0}(K)
\end{array}\right.
\end{aligned}
$$

Evidemment, d'autres choix sont possibles. Nous référons à [6] pour d'autres éléments finis. Par exemple, on peut considérer l'approximation du problème de Stokes (3) par $P_{2}-P_{0}$ (i.e., la vitesse est approximée par $P_{2}$ continue et la pression par $P_{0}$ ) ce qui conduit à l'élément fini de plus bas degré de Fraeijs de Veubeke [7]. Cependant, pour cet élément fini on a une perte d'un ordre dans les estimations d'erreurs à cause de l'approximation faible de la pression (et par conséquent de $\omega$ ). Un autre choix possible est de considérer l'approximation du problème de Stokes (3) par $P_{2} \oplus b_{K}$ continue pour la vitesse et $P_{1}$ continue pour la pression. Ceci conduit à un élément fini similaire à l'élément PEERS mais avec un ordre de convergence plus élevé. On peut faire la même construction avec des méthodes d'ordres plus élevés et aussi avec des quadrilatères. Nous référons à [6] pour d'autres éléments finis et leurs analyses.

CONCLUSION. Une approche unifiée de construction d'éléments finis pour le problème de l'élasticité linéaire, basée sur une formulation dualehybride, a été présentée. Cette construction est reliée à l'approximation du problème de Stokes en variables primitives et elle conduit à de nouveaux éléments finis. L'élément fini PEERS est celui de plus bas degré (avec le minimum de degrés de liberté) mais la variable correspondante au rotationnel ne peut être éliminée élément par élément puisqu'elle est continue. Dans le but d'aboutir à des approximations constantes par morceaux pour les 
multiplicateurs de Lagrange et la pression en tant que seules inconnues du système discret, nous pensons que nous avons à chercher un élément fini nonconforme.

\section{REFERENCES}

[1] M. AmARA and J.M. Thomas. Equilibrium finite elements for the linear elastic problem. Numer. Math. 33, pp. 367-383 (1979).

[2] D.N. Arnold, F. Brezzi and J. Douglas. PEERS: A new mixed finite element for plane elasticity. Japan J. Appl. Math. 1, pp. 347-367 (1984).

[3] F. Brezzi and M. Fortin. Mixed and Hybrid Finite Element Methods. SpringerVerlag, Berlin, New York, 1991.

[4] M. Crouzeix and P.A. Raviart. Conforming and nonconforming finite element methods for solving the stationary Stokes equations. RAIRO, Anal. Numer. 3, pp. 33-76 (1973).

[5] M. FARHLOUL and M. ForTIN. A new mixed finite element for the Stokes and elasticity problems. SIAM J. Numer. Anal. 4, pp. 971-990 (1993).

[6] M. FARHLOUL and M. Fortin. Dual hybrid methods for the elasticity and the Stokes Problems: A unified approach. To appear in Numer. Math.

[7] B.X. FraeiJs DE VEubeKE. Stress function approach. World Congress on the finite element method in structural mechanics, Bournemouth, Dorest, England (1975).

[8] V. GiRault and P.A. Raviart. Finite Element for Navier-Stokes Equations, Theory and Algorithms. Springer S.C.M. 5, Springer-Verlag, 1986.

[9] P. HoOD and C. TAYLOR. A numerical solution of the Navier-Stokes equations using the finite element technique. Comput. Fluids 1, pp. 73-100 (1973).

[10] P.A. Raviart and J.M. Thomas. A mixed finite element method for 2nd order elliptic problems. Lecture Notes in Math., 606, Springer-Verlag, Berlin, New York, pp. 292315 (1977).

[11] R. Stenberg. A family of mixed finite elements for the elasticity problem. Numer. Math. 53, pp. 513-538 (1988).

[12] J.M. Thomas. Sur l'analyse numérique des méthodes d'éléments finis hybrides et mixtes. Thèse, Université Pierre et Marie Curie, Paris 6, 1977.

Département de mathématiques et de statistique, Université de Moncton, Moncton, NB, E1A 3E9 Canada

Département de mathématiques et de statistique, Université Laval, Québec, QuÉ., G1K 7 P4 CANADA 